ORIGINAL ARTICLE

\title{
Cross national study of injury and social determinants in adolescents
}

\author{
W Pickett, M Molcho, K Simpson, I Janssen, E Kuntsche, J Mazur, Y Harel, W F Boyce
}

Injury Prevention 2005;11:213-218. doi: 10.1136/ip.2004.007021

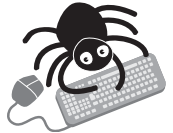

Tables 2 to 6 can be viewed on our website.

See end of article for authors' affiliations

........................

Correspondence to: Dr William Pickett, Department of Community Health and Epidemiology, Queen's University, Angada 3, 76 Stuart Street, Kingston General Hospital, Kingston, Ontario, Canada, K7L 3N6; pickettw@ post.queensu.ca
Objectives: To compare estimates of the prevalence of injury among adolescents in 35 countries, and to examine the consistency of associations cross nationally between socioeconomic status then drunkenness and the occurrence of adolescent injury.

Design: Cross sectional surveys were obtained from national samples of students in 35 countries. Eight countries asked supplemental questions about injury.

Setting: Surveys administered in classrooms.

Subjects: Consenting students ( $n=146440$; average ages 11-15 years) in sampled classrooms. 37878 students (eight countries) provided supplemental injury data.

Exposure measures: Socioeconomic status (material wealth, poverty) and social risk taking (drunkenness). Outcome measures: Specific types and locations of medically treated injury.

Results: By country, reports of medically treated injuries ranged from 33\% (1060/3173) to 64\% (1811/ 2833 ) of boys and $23 \%(740 / 3172)$ to $51 \%$ (1485/2929) of girls, annually. Sports and recreation were the most common activities associated with injury. High material wealth was positively $(O R>1.0 ; p<0.05)$ and consistently (6/8 countries) associated with medically treated and sports related injuries. Poverty was positively associated with fighting injuries (6/8 countries). Drunkenness (social risk taking) was positively $(p<0.01)$ and consistently (8/8 countries) associated with medically treated, street, and fighting injuries, but not school and sports related injuries.

Conclusion: The high prevalence of adolescent injury confirms its importance as a health problem. Social gradients in risk for adolescent injury were illustrated cross nationally for some but not all types of adolescent injury. These gradients were most evident when the etiologies of specific types of adolescent injury were examined. Prevention initiatives should focus upon the etiologies of specific injury types, as well as risk oriented social contexts.
O ver the past two decades, injury has been recognized as an important cause of morbidity in pediatric populations and is a leading cause of death among children internationally. ${ }^{1-3}$ In many countries ${ }^{14}$ pediatric injuries account for more childhood deaths than all other causes combined. Burdens associated with pediatric injury in terms of lost potential, disability, treatment costs, and rehabilitation are substantial. ${ }^{45}$ Peaks in the occurrence of injury related traumas are observed in adolescents ${ }^{1}$ and their etiologies are obvious health research priorities. ${ }^{6}$ Social risk factors are of interest as potential injury determinants, yet studies of social factors and adolescent injury are uncommon, especially cross nationally.

Increasing attention has been given to the effects of socioeconomic status on health, although research in the injury field has mainly concentrated on adults ${ }^{78}$ and young children. ${ }^{9}{ }^{10}$ Socioeconomic status has been shown to be negatively associated with injury risk but the level of association varies from modest ${ }^{7112}$ to strong ${ }^{781314}$ according to the type of injury, the population under study, and the indicators of socioeconomic status that were applied. Few studies of adolescents have examined socioeconomic status relations with specific types of injury, and existing studies report inconsistent findings. ${ }^{15}{ }^{16}$ This aspect of the social aetiology of injury is of continued interest as the economic gap between the rich and poor is widening ${ }^{17}$ and adolescents who are financially deprived may be embedded in a hazardous social context.

Risk taking behaviour represents a second potential focus for etiological studies. ${ }^{18}$ Adolescent lifestyles that include engagement in substance use, truancy, and the taking of physical and sexual risks are the social norm in many adolescent societies. ${ }^{19-21}$ Most of these risk behaviours (for example, drunkenness ${ }^{22-24}$ ) occur in a social context. A recent systematic review ${ }^{25}$ that examined the link between social risk taking and injury identified few $(\mathrm{n}=7)^{26-32}$ high quality studies that focused upon these topics, and none among adolescents. Risk taking behaviour was associated with the occurrence of unintentional injury except in the case of injuries in highly skilled sports.

In this study, we present contemporary estimates of the prevalence of injury among adolescents in 35 countries. We also examine relations between illustrative social determinants (two measures of socioeconomic status; drunkenness, a risk behaviour that occurs in social contexts $\mathrm{s}^{22-24}$ ) and the occurrence of specific types of adolescent injury (school, street, sports related, and fighting). The etiologic analysis was used to model the occurrence of different types of injury (dependent variables) as a function of socioeconomic status and drunkenness (independent variables), in multiple logistic regression analyses that adjusted for other known risk factors. Through this analysis we hoped to: (1) describe contemporary rates of adolescent injury on a cross national basis; (2) use illustrative examples of social factors and specific types of injury to confirm the existence of gradients in risk for injury when the social etiologies of specific (for example, sports, fighting) as opposed to general (for example, injured/not injured) injury outcomes were examined, and (3) determine whether these gradients could be

Abbreviations: HBSC, Health Behaviour in School-Aged Children survey. 
observed consistently across countries. Increased understanding of the distribution of the injury problem as well as potential social determinants could guide future preventive interventions.

\section{METHODS}

\section{Description of survey and study population}

The study was based on international records from the 2001/ 2002 Health Behaviour in School-Aged Children survey (HBSC); a World Health Organization collaborative cross national study. The HBSC involves a cross sectional survey of elementary and high school students. ${ }^{33}$ The survey consists of a questionnaire completed in the classroom (completion time 45 minutes). Institutional ethics approval is obtained at the country level.

The goal of the HBSC is to identify youth health indicators and the factors that influence them. ${ }^{33}$ One of its primary functions is to further understanding about the social factors that put adolescents at risk or are notably protective. The survey includes a set of indicators that provide a valid representation of the health and lifestyle of adolescents in industrialized countries.

\section{Sampling}

A full description of the sampling method appears in the HBSC protocol (see http://www.hbsc.org). Because differences exist between countries in "school systems, age of admission to school and levels of advancement of students across countries", the HBSC cannot have a uniform approach to sampling. ${ }^{33}$ The sampling protocol always involves a cluster design with the school class being the basic cluster. Countries are initially asked to randomly sample schools that teach 11-15 year old children. School classes are then selected using a weighted probability technique to ensure that students from schools of different sizes are equally likely to be included. In some countries, regional geography and other salient demographic factors (for example, religion, language of instruction) are taken into consideration via stratification. ${ }^{33}$

\section{Measures}

The 2001/2002 HBSC survey contained both mandatory items and optional groups of questions. This analysis focused upon injury items (one mandatory question; an optional package of items used in eight countries), as well as available measures of social risk factors: socioeconomic status items (two mandatory questions), and a drunkenness item (one mandatory question). All measures were taken from previous studies, $^{34}{ }^{35}$ previous HBSC surveys, ${ }^{34}$ or were adapted from existing surveillance initiatives. ${ }^{36}{ }^{37}$ All measures were pretested for face and content validity in classrooms within each participating country.

\section{Outcome measures: injury}

The occurrence of injury was measured using a single mandatory question that is consistent with the widely accepted definition of an injury as an "event that requires medical attention". ${ }^{33}{ }^{35}$ Questions about injury were preceded with the following statement: "Many young people get hurt or injured from activities such as playing sports or fighting with others at different places such as the street or home. Injuries can include being poisoned or burned. Injury does not include illnesses such as Measles or the Flu. The following questions are about injuries you may have had during the past 12 months." Participants were asked to report injury events that required medical attention from a doctor or a nurse. Response options were: "I was not injured in the past 12 months"; "one time"; "two times"; "three times"; "four or more times".
Eight countries employed the optional package of injury questions: Belgium-French, Canada, Czech Republic, Estonia, Israel, Latvia, Poland, and the United States (high school students only). Participants reporting one or more medically treated injuries were asked to identify their most serious injury event. Supplemental items included close-ended questions that addressed external causes of injury. This was done because "virtually all injury classification systems s $^{36} 37$ attempt to describe and classify the external factors and circumstances that precipitate injury events". ${ }^{34} 38$ Descriptors of external cause included: location of injury (response options included: "home or yard"; "school including school grounds"; "sports facilities or fields"; "street, road or parking $l^{\prime \prime} t^{\prime \prime}$ and the mechanism of injury (response options included but not limited to: "playing or training for organized sport or recreational activity"; "physical fight"). The wording of these questions and the response categories was based upon existing surveillance systems ${ }^{36}{ }^{37}$ but limited to common responses observed in previous surveys. ${ }^{34} 39$

\section{Primary risk factor variables}

A composite Family Affluence Scale score was calculated based on responses to questions about family, as follows: "car ownership"; "owning a bedroom for oneself"; "holiday travel during past 12 months"; "computer ownership". This scale infers absolute levels of material wealth expressed in terms of consumption, has a possible range of values from $0-7$, is conceptually related to common indices of material deprivation, ${ }^{39} 40$ and is similar to an existing index of home affluence. ${ }^{41}$ The motivation for inclusion of this variable was to consider the relative effects of deprivation and wealth on injury risk.

A more direct measure of poverty was also derived from a question reporting the frequency that a young person went to school or bed hungry because there is not enough food at home (response options: "always"; "often"; "sometimes"; "never").

As a measure of social risk taking, participants reported the number of times that they had ever consumed so much alcohol that they were really drunk (response options: "never"; "once"; "4-10 times"; "more than 10 times"). This drunkenness measure has been used in the HBSC survey since its inception, and represents a commonly used, validated approach to the assessment of heavy alcohol intake on single occasions. ${ }^{42}$ It has proven to be a "very good predictor" of other risk behaviours, as well as poor adjustment to school. ${ }^{33}$

\section{Confounding variables}

Three variables were selected a priori for inclusion as confounders: age (in years), sex, and physical activity participation. These variables are risk factors for injury, and preliminary analyses demonstrated variations in these variables by the social risk factors under study.

Physical activity was measured as follows: after being provided with a definition and examples of common activities, subjects were asked how many days in the past week and in a typical week they were physically active (cumulative activity) for 60 minutes or more. These questions were based on the Moderate-to-Vigorous Physical Activity measure developed by Prochaska and colleagues. ${ }^{43}$ The mean number of days from the past week and a typical week were used as an index of participation. ${ }^{43}$

\section{Statistical analysis}

Descriptive analyses were conducted within each country to obtain the prevalence of medically treated injury by sex, and these were ranked across the 35 countries. Frequency distributions of injury occurrence were plotted within the 
eight countries by location then mechanism of injury. Multiple logistic regression analyses were used to estimate odds ratios (OR) for injury associated with the available social risk factors (material wealth, poverty, drunkenness). Separate models for eight countries were run for injury locations (for example, school injuries, home injuries, street injuries), and mechanisms (for example, playing or training for sports/recreational activities, fighting injuries). All odds ratio estimates were adjusted simultaneously for age, sex, physical activity, and the other primary social risk factors. All descriptive and regression analyses were performed within countries and were conducted using SPSS (version 12, SPSS Inc, Chicago, IL, USA). Design effects of 1.20 were applied to standard errors and hence confidence intervals surrounding risk estimates using Excel (version 2000, Microsoft Inc, Redmond, WA, USA).

\section{RESULTS}

\section{Samples}

146440 children in 35 countries provided reports of injury experiences and of these, 38878 children in eight countries answered supplemental questions about their one most serious injury (if any). At the participant level, responses rates varied from $64.5 \%$ (3481/5400) in Latvia to $91.2 \%$ (4560/ 5000) in Estonia.

\section{Descriptive findings}

The percentage of youth reporting one or more medically treated injuries within the past 12 months was more common in boys than in girls in all 35 countries, with cross national prevalence estimates ranging from 33\% (1060/3173) to $64 \%(1811 / 2833)$ of boys and $23 \%(740 / 3172)$ to $51 \%$ (1485/2929) of girls (fig 1). Estimates of youth reporting a single injury were much less variable. Major differences in prevalence estimates by country were attributable to variations in the number of youth reporting multiple injuries. There was no evidence of strong regional (for example, North America $v$ Europe) differences.

Among the eight countries that used optional injury questions, the median proportions of youth reporting one or more injuries were very high (table 1). There was considerable variation between countries in the rank order of injury locations. By activity, playing or training for a sport/ recreational activity was the leading external cause of injury. Fighting injury was the only form of intentional injury reported. Summary values provided in table 1 indicate the variability between countries in the prevalence of social risk factors, by sex, as well as median ages and levels of physical activity of the samples of adolescents under study.

\section{Etiologic findings \\ Medically treated injuries}

Low material wealth was protective for the occurrence of medically treated injuries (6/8 countries), with the suggestion of a protective effect in the other two countries (see http://www.injuryprevention.com/supplemental for table 2). Always/often going to bed or school hungry (an indicator of poverty) was an uncommon social exposure, but as inferred from point estimates it was consistently (6/8 countries) associated with trends towards increased risk for medically treated injuries. Frequent drunkenness during a youth's lifetime was a strong and consistent risk factor for medically treated injuries ( $8 / 8$ countries).

\section{School injuries}

The occurrence of school injuries was not consistently associated with low levels of material wealth, going to bed or school hungry, or drunkenness (see http://www.injuryprevention. com/supplemental for table 3 ).

\section{Sports-related injuries}

Low material wealth was strongly and consistently associated with protection from sports related injuries, even after controlling for physical activity (8/8 countries; see http:// www.injuryprevention.com/supplemental for table 4). There was no evidence of associations between poverty and sports related injury, and inconsistency in the risk estimates observed for frequent drunkenness and sports related injury.

\section{Street injuries}

There was no evidence of significant associations between material wealth and street injuries (0/8 countries; see http:// www.injuryprevention.com/supplemental for table 5). The direction of risk estimates for poverty/hunger and street injury were positive but not always statistically significant. Frequent drunkenness was a strong and consistent risk factor for street injuries ( $8 / 8$ countries), and the risk estimates generally followed a dose related pattern.

\section{Fighting injuries}

Fighting injuries were uncommon and this is reflected in the variability of the confidence intervals surrounding risk estimates (see http://www.injuryprevention.com/supplemental for table 6). There was no consistent association between low material wealth and fighting injuries. Although not statistically significant, based upon the consistency of point estimates (6/8 countries) there was the suggestion of an association between poverty/hunger and fighting injuries. Drunkenness was a strong and significant risk factor for fighting injuries (8/8 countries).

\section{DISCUSSION}

With a prevalence between $33 \%$ and $62 \%$ across countries among males (19\% to $39 \%$ among females), our findings indicate that adolescent injuries are very common cross nationally. Our analysis represents one of the first cross national examinations of adolescent injury patterns, and our focus on the social etiology of specific types of injury cross nationally is also novel. Variations in the strength and direction of associations were observed for different combinations of social risk factors and types of injury. Increased material wealth was positively associated with sports related but none of the other forms of injury. Drunkenness (as a measure of socially oriented risk taking) and hunger (as a measure of poverty) were positively and consistently associated with street injuries and injuries involving physical fights, but not school and sports related injuries. These examples do illustrate that specific types of adolescent injury appear to have different social etiologies.

The cross national variations in injury prevalence observed are intriguing, but it is unclear whether these variations are attributable to underlying differences in risk. Our analysis involved study of medically treated injuries, and there are known international variations in access to medical treatment. ${ }^{44}$ The 35 countries under study operate under a variety of healthcare delivery models from fully public systems to systems that mix public and private healthcare models. ${ }^{45}$ This may in part account for the apparent protective effect of low material wealth on rates of medically treated injury and sports injury. Some but not all countries have resident nurses in school systems. This too could contribute to overreporting of injury events in more affluent schools, which would introduce a differential bias by socioeconomic class.

Depending upon the country, the 2001/2002 HBSC was administered temporally during fall or winter school terms. Risks for adolescent injury vary by season, and injuries are most reliably reported within three months. ${ }^{46}$ Variations in the timing of the survey across countries may have impacted injury rates and hence the cross national comparisons. 

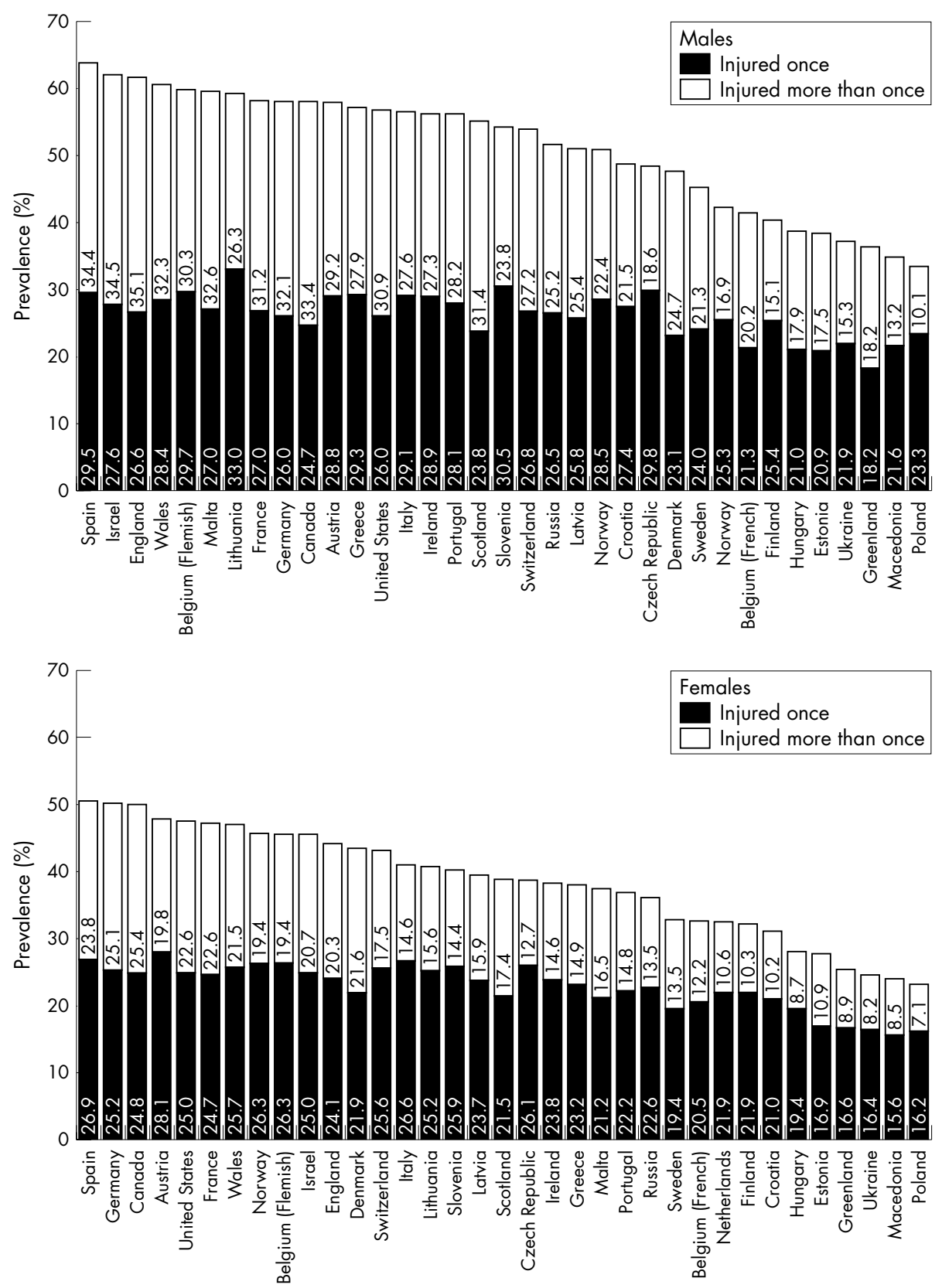

Figure 1 Annual prevalence of medically treated injuries among 146440 adolescents in 35 countries, by sex.

With respect to etiologic findings, past studies of socioeconomic status and injury have demonstrated consistent gradients in risk for injury in association with low socioeconomic status among general populations, ${ }^{78}$ populations of young children, ${ }^{910}$ and for specific types of injury. ${ }^{47}$ Most existing studies have focused upon fatal or other forms of traumatic injury, and ecological (group level) analyses predominate this literature. Social gradients in risk for injury have not, however, been demonstrated consistently within adolescent populations. ${ }^{15}{ }^{16} 48$ Past explanations for this issue centre around the assessment of socioeconomic status in adolescents, and the unique mixing that occurs in adolescent cultures. ${ }^{151648}$ Our study confirms that gradients in adolescent injury risks by socioeconomic class do exist, but are only clearly demonstrated when specific combinations of socioeconomic status measures and injury types are examined in a focused manner.
Our measure of poverty was consistently associated with injuries caused by violence (fighting) and injuries in less organized (street/road) locations. Mechanistically, this poverty indicator appears to be operating as a measure of social deprivation and these associations demonstrate the existence of classic, inverse health gradients among adolescent populations that are exposed to contextual risks. Poorer children may not be afforded protection from physical risks in their social environment. Similar risk gradients were not identified in school locations which suggests that social context does impact upon health risk gradients.

We failed to identify associations between the Family Affluence Scale and most external causes of injury. Exceptions were associations between family affluence and sports/recreational injuries, which were in a positive direction (higher affluence, more injuries). The family affluence measure may correlate strongly with increased opportunities 


\begin{tabular}{|c|c|c|c|c|c|c|}
\hline & \multicolumn{6}{|c|}{ Estimates* for eight countries } \\
\hline & \multicolumn{3}{|l|}{ Males } & \multicolumn{3}{|l|}{ Females } \\
\hline & Median & Min & $\operatorname{Max}$ & Median & Min & Max \\
\hline $\begin{array}{l}\text { Medically treated injury in the past } 12 \text { months (\%) } \\
\text { By location (\% of injured) }\end{array}$ & 49.8 & 33.4 & 62.1 & 39.2 & 23.3 & 50.1 \\
\hline Home or yard & 20.8 & 11.6 & 36.3 & 28.5 & 19.1 & 38.7 \\
\hline School or school grounds & 22.8 & 14.4 & 32.4 & 23.1 & 16.2 & 37.9 \\
\hline Sports facilities or field & 25.0 & 15.7 & 34.1 & 15.5 & 6.5 & 30.1 \\
\hline Street, road, or parking lot & 11.2 & 8.0 & 18.8 & 10.2 & 5.5 & 15.1 \\
\hline $\begin{array}{l}\text { By activity (\% of injured) } \\
\text { Playing or training for a sport or recreational } \\
\text { activity }\end{array}$ & 40.5 & 31.9 & 55.4 & 37.0 & 19.0 & 59.1 \\
\hline Physical fight & 7.2 & 4.2 & 8.1 & 3.6 & 1.7 & 4.7 \\
\hline \multicolumn{7}{|l|}{ Primary social risk factors } \\
\hline \multicolumn{7}{|l|}{ Socio-economic status } \\
\hline Family Affluence Scale (FAS): (\% low: (FAS 0-3)) & 30.3 & 10.3 & 49.6 & 36.5 & 10.9 & 61.5 \\
\hline Go to bed or school hungry (\% always/often) & 2.9 & 2.3 & 6.9 & 1.9 & 1.1 & 3.5 \\
\hline Social behaviour & & & & & & \\
\hline Drunkenness in lifetime (\% 4 or more times) & 7.8 & 4.6 & 15.9 & 4.2 & 1.5 & 9.1 \\
\hline \multicolumn{7}{|l|}{ A priori confounders } \\
\hline Age (years) & 13 & 10 & 17 & 13 & 10 & 17 \\
\hline Weekly physical activity (mean days per week) & 4.0 & 3.5 & 5.0 & 3.5 & 3.0 & 4.0 \\
\hline
\end{tabular}

for participation in organized sports that are expensive. We also recognize the potential for a differential misclassification of sports injury outcomes by level of material wealth due to variations in access to medical care.

Strong associations were observed between increased amounts of drunkenness and fighting injuries and injuries that occurred on the street. Drunkenness is clearly a direct cause of injury, and its association with some but not all injury outcomes is expected given variations in social context that surround adolescent drinking patterns. Drunkenness is also a marker for a problem behaviour lifestyle ${ }^{18}$ that includes engagement in multiple risk behaviour. ${ }^{48}{ }^{49}$ This social behaviour is normative in many youth cultures, and is associated with a variety of injury consequences. It is also notable that similar etiologic patterns exist for fighting and street injuries in that poverty and drunkenness were associated with these injury types. This pattern might suggest that aggression is also a mediating factor for certain types of injury that occur in unstructured social contexts.

Major strengths of this analysis are its international scope and the large numbers of young people involved from a diversity of cultures. The HBSC questionnaire items were developed iteratively over several years and are supported by validation efforts. ${ }^{33}$ The ability to examine relations between different social measures and a variety of injury types is a second strength. Gradients in risk for injury may be masked in other studies that examine more general injury outcomes. Weaknesses include the fact that data were based upon self reports with associated reporting errors, and inferences made about etiology are further limited by the cross sectional nature of data collection. In addition, we studied only a limited range of social risk factors and injury types. In the future it would be better to study a broader range of risk factors in a prospective manner using validated measures that were not reliant on self-reports. This task would be very difficult to accomplish on a cross national basis.

Our findings inform the planning of future social research. There is an inherent need for etiologic models to be theoretically based, and there is also a need for injury studies to focus upon underlying physical mechanisms (for example, impairment, hazards) and behavioral mechanisms (for example, bullying, aggression) that directly link social risk factors to injury occurrence. These mechanisms are likely to be responsible for the fact that individual risk factor measures are associated with some but not all injury outcomes. Some existing studies of socioeconomic status and injury, for example, use only a single measure of socioeconomic status and do not consider the possibility that etiologic relations may vary by injury type. In addition, characteristics of social contexts (home, school, peer group) that could protect adolescents from injury, or conversely put them at increased risk, also require consideration. Conceptual models such as the population health framework ${ }^{50}$ could be useful in that regard. This framework states that determinants of health operate at two levels-contextual and individual. With respect to the etiology of adolescent injury, contextual and individual determinants may have direct and additive effects that would interact to produce varying levels of risk..$^{50}$

The results also have implications for adolescent health policy. Adolescent injuries should be recognized as a major public health issue. Social gradients in risk for adolescent injury can be demonstrated cross nationally for some but not all types of adolescent injury. These gradients are most evident when the etiologies of specific types of adolescent injury are examined. Health policy surrounding the

\section{Key points}

- The social etiology of adolescent injury remains poorly understood.

- This cross national study demonstrates that injury is an important, contemporary adolescent health problem cross nationally.

- Gradients in risk for adolescent injury can be demonstrated for some combinations of social risk factors and injury. These are evident when the etiologies of specific as opposed to general forms of adolescent injury are examined.

- Some social contexts appear to protect adolescents from socially oriented injury events. 
prevention of adolescent injuries requires a solid evidence base, and our findings identify vulnerable subgroups and social contexts that lead consistently to the occurrence of adolescent injury. Preventive interventions should also focus upon the etiologies of specific as opposed to general injury types, as well as these risk oriented social contexts.

\section{ACKNOWLEDGEMENTS}

Please visit http://www.injuryprevention.com/supplemental for the full list of acknowledgements.

\section{Authors' affiliations}

W Pickett, K Simpson, I Janssen, W F Boyce, Department of Community Health and Epidemiology, Queen's University, Kingston, Canada W Pickett, K Simpson, Department of Emergency Medicine, Queen's University, Kingston, Canada

M Molcho, Y Harel, Department of Sociology and Anthropology, BarIllan University, Ramat Gam, Israel

I Janssen, School of Physical and Health Education, Queen's University, Kingston, Canada

E Kuntsche, Swiss Institute for the Prevention of Alcohol and Drug Problems, Lausanne, Switzerland

J Mazur, Mother and Child National Research Institute, Warsaw, Poland W F Boyce, Social Program Evaluation Group, Faculty of Education, Queen's University, Kingston, Canada

\section{REFERENCES}

1 Health Canada. Leading causes of death and hospitalization in Canada. Available at http://www.hc-sc.gc.ca/pphb-dgspsp/publicat/lcd-pcd97/ mrt_mf_e.html\#per (accessed 1 August 2004).

2 Choinière $R$, Robitaille $Y$. Methodological considerations and overall profile of mortality, hospitalizations and emergency room visits. In: Beaulne $G$, ed. For the safety of Canadian children and youth: from injury data to preventive measures. Ottawa, ON: Minister of Public Work and Government Services Canada, 1997:11-36. Catalogue No. H39-412/1997E.

3 Danseco ER, Miller TR, Spicer RS. Incidence and costs of 1987-1994 childhood injuries: demographic breakdowns. Pediatrics 2000;105:E27.

4 Krug EG, Sharma GK, Lozano R. The global burden of injuries. Am J Public Health 2000;90:523-6.

5 Angus DE, Cloutier E, Albert T, et al. The economic burden of unintentional injury in Canada. Toronto, ON: SMARTRISK, 1998:46.

6 Morongiello B. Unintentional injury prevention-priorities for research, capacity building, and knowledge translation. Guelph, ON: University of Guelph, 2003:1-16

7 Black D, Morris J, Smith C, et al. Inequalities in health. The Black Report. Markham: Penguin Books, 1982:126-34.

8 Cubbin C, LeClere FB, Smith GS. Socioeconomic status and injury mortality individual and neighbourhood determinants. J Epidemiol Community Health 2000;54:517-24.

9 Reading R, Langford IH, Haynes R, et al. Accidents to preschool children: comparing family and neighbourhood risk factors. Soc Sci Med 1999;48:321-30

10 Haynes R, Reading R, Gale S. Household and neighbourhood risks for injury to 5-14 year old children. Soc Sci Med 2003;57:625-36.

11 Mare RD. Socioeconomic effects on child mortality in the United States. Am J Public Health 1982;72:539-47

12 Roberts I. Cause specific social class mortality differentials for child injury and poisoning in England and Wales. J Epidemiol Community Health 1997:51:334-5.

13 Cubbin C, LeClere FB, Smith GS. Socioeconomic status and the occurrence of fatal and nonfatal injury in the United States. Am J Public Health 2000;90:70-7.

14 Jolly DL, Moller JN, Volkmer RE. The socio-economic context of child injury in Australia. J Paediatr Child Health 1993;29:438-44.

15 Williams JM, Currie CE, Wright $P$, et al. Socioeconomic status and adolescent injuries. Soc Sci Med 1997;44:1881-91.

16 Faelker T, Pickett W, Brison RJ. Socioeconomic differences in childhood injury: a population based epidemiologic study in Ontario, Canada. Inj Prev 2000;6:203-8

17 Mackenbach JP, Bos $\mathrm{V}$, Andersen $\mathrm{O}$, et al. Widening socioeconomic inequalities in mortality in sex Western European countries. Int J Epidemiol 2003:32:830-7.

18 Jessor R. Risk behavior in adolescence: a psychosocical framework for understanding and action. J Adolesc Health 1991;12:597-605.

19 Spirito A, Rasile DA, Vinnick LA, et al. Relationship between substance use and self-reported injuries among adolescents. J Adolesc Health 1997;21:221-4.
20 Dryfoos JG. Adolescents at risk: a summation of work in the field-programs and policies. J Adolesc Health 1991;12:630-7.

21 Sells WC, Blum RW. Current trends in adolescent health. In: DiClemente RJ, Hansen WB, Ponton LE, eds. Handbook of adolescent health risk behavior New York: Plenum Press, 1996:5-34.

22 Christiansen M, Vik PW, Jarchow A. College student heavy drinking in social contexts versus alone. Addict Behav 2002;27:393-404

23 Kuntsche EN, Gmel G. Emotional well-being and violence among social and solitary risky single occasion drinkers in adolescence. Addiction 2004;99:331-9.

24 Kuntsche E, Rehm J, Gmel G. Characteristics of binge drinkers in Europe. Soc Sci Med 2004;59:113-27.

25 Turner C, McClure R, Pirozzo S. Injury and risk-taking behavior-a systematic review. Accid Anal Prev 2004;36:93-101.

26 Crutcher JC, Black G, Campbell P, et al. Risky driving behaviors among teenagers - Gwinnett County, Georgia, 1993. Morb Mortal Wkly Rep 1994;43:405-9.

27 Perneger T, Smith GS. The driver's role in fatal two-car crashes: a paired "case-control" study. Am J Epidemiol 1991;134:1138-45.

28 Rajalin S. The connection between risky driving and involvement in fatal accidents. Accid Anal Prev 1994;26:555-62.

29 Bell NS, Amoroso PJ, Yore MM, et al. Self-reported risk-taking behaviors and hospitalization for motor vehicle injury among active duty army personnel. Am J Prev Med 2000;18(Suppl 3):85-95.

30 Mawson AR, Jacobs KW, Winchester $Y$, et al. Sensation-seeking and traumatic spinal cord injury: a case-control study. Arch Phys Med Rehabil 1988;69:1039-43.

31 Cherpitel CJ, Meyers AR, Perrine MW. Alcohol consumption, sensation seeking and ski injury: a case-control study. J Stud Alcohol 1998:59:216-21.

32 Bouter LM, Knipschild PG, Feij JA, et al. Sensation seeking and injury risk in downhill skiing. Pers Individ Dif 1998;9:667-73.

33 Currie C, Samdal O, Boyce W, et al. Health Behaviour in School-aged Children: a WHO cross-national study. Research protocol for the 2001/2002 survey. Edinburgh, UK: University of Edinburgh, Child and Adolescent Health Research Unit, 2001:2-37; 28-37; 82; 111-15.

34 Currie CE, ed. Health Behaviour in School-aged Children: research protocol for the 1997-98 survey. Edinburgh, UK: WHO Coordinating Center for the Study of Health Behaviour in School-Aged Children, 1998:2-11.

35 Christoffel KK, Scheidt PC, Agran PF, et al. Standard definitions for childhood injury research: excerpts of a conference report. Pediatrics 1992;89:1027-34

36 WHO Working Group on Injury Surveillance Methods. International Classification of External Causes of Injuries (ICECI): data dictionary, version 1.1a. Adelaide: Consumer Safety Institute, Amsterdam and AlHW National Injury Surveillance Unit, 2003.

37 Bay-Nielsen H. NOMESCO classification of external causes of injury. Copenhagen: Nordic Medico-Statistical Committee, 1997.

38 Centres for Disease Control and Prevention, National Center for Health Statistics. Proceedings of the International Collaborative Effort on Injury Statistics, Vol 1. Bethesda, MD: DHHS Publication no (PHS) 95-1252, 1994.

39 Currie CE, Elton RA, Todd J, et al. Indicators of socioeconomic status for adolescents: the WHO Health Behaviour in School-aged Children Survey. Health Educ Res 1997:12:385-97.

40 Torsheim T, Currie C, Boyce W, et al. Material deprivation and self-rated health: a multilevel study of adolescents from 22 European and North American countries. Soc Sci Med 2004;59:1-12.

41 Wardle J, Robb K, Johnson F. Assessing socioeconomic status in adolescents: the validity of a home affluence scale. J Epidemiol Community Health 2002;56:595-9.

42 Gmel G, Rehm J, Kuntsche E. Binge drinking: definitions, epidemiology, trends, and consequences. Sucht 2003:49:105-16.

43 Prochaska JJ, Sallis JF, Long B. A physical activity screening measure for use with adolescents in primary care. Arch Pediatr Adolesc Med 2001;155:554-9.

44 Schoen C, Doty MM. Inequities in access to medical care in five countries: findings from the 2001 Commonwealth Fund International Health Policy Survey. Health Policy 2004;67:309-22.

45 Rosenthal MM. Systems, strategies, and some patient encounters: a discussion of twelve countries. In: Rosenthal MM, Frenkel M, eds. Health care systems and their patients: an international perspective. Boulder: Westview Press, 1992:329-45.

46 Harel Y, Overpeck MD, Jones DH, et al. The effect of recall on estimating annual nonfatal injury rates for children and adolescents. Am J Public Health 1994;84:599-605.

47 Dougherty G, Pless IB, Wilkins R. Social class and the occurrence of traffic injuries and deaths in urban children. Can J Public Health 1990:81:204-9.

48 Pickett W, Schmid H, Boyce WF, et al. Multiple risk behaviours and injury: an international study of youth in 12 countries. Arch Pediatr Adolesc Med 2002; 156:786-93.

49 Pickett W, Garner MJ, Boyce WF, et al. Gradients in risk for youth injury associated with multiple-risk behaviors: a study of 11,329 Canadian adolescents. Soc Sci Med 2002;55:1055-68.

50 Federal, Provincial and Territorial Advisory Committee on Public Health Strategies for population health: investing in the health of Canadians. Ottawa, ON: Minister of Supply and Services Canada, 1994:28-39. Catologue No H39-316/1994E. 